\title{
Electron Attachment in Ice-HCl Clusters: An Ab Initio Study
}

\author{
Xifeng Li and Léon Sanche, \\ Département de Médecine Nucléaire et de Radiobiologie, \\ Faculté de Médecine, Universite de Sherbrooke \\ and \\ Arvi Rauk and David Armstrong*, \\ Department of Chemistry, \\ University of Calgary \\ Supplementary Data
}

- corresponding author: armstron@ucalgary.ca 
Table S1 Dipole moments, relative energies and B3LYP electron binding energies for $\left(\mathrm{H}_{2} \mathrm{O}\right)_{6}$ and $\left(\mathrm{H}_{2} \mathrm{O}\right)_{12}$ neutral systems.

\begin{tabular}{ccccc}
\hline $\begin{array}{c}\text { Neutral Ice } \\
\text { system }\end{array}$ & $\begin{array}{c}\text { Figure with } \\
\text { structure }\end{array}$ & $\begin{array}{c}\text { Dipole/ } \\
(\mathrm{D})\end{array}$ & $\begin{array}{c}\text { Energy/ } \\
\mathrm{kJ} \mathrm{mol}^{-1}\end{array}$ & $\begin{array}{c}\mathrm{EBE} / \\
\mathrm{eV}\end{array}$ \\
\hline$\left(\mathrm{H}_{2} \mathrm{O}\right)_{12}$ (hexagonal) & $1(\mathrm{a} 1, \mathrm{a} 2)$ & 6.0 & 64 & 0.49 \\
$\left(\mathrm{H}_{2} \mathrm{O}\right)_{12}$ (upper layer opt'd) & $2(\mathrm{a})$ & 4.2 & 0 & 0.31 \\
$\left(\mathrm{H}_{2} \mathrm{O}\right)_{6}$ (hexagonal) & $2(\mathrm{c})$ & 4.4 & 39 & 0.22 \\
$\left(\mathrm{H}_{2} \mathrm{O}\right)_{6}$ (amorphous) & 2(b) & 0.58 & 0 & -0.04 \\
\hline
\end{tabular}


Table S2: Coordinates of $\mathrm{H}^{\bullet} \ldots \mathrm{Cl}^{-} .\left(\mathrm{H}_{2} \mathrm{O}\right)_{12}$ complex from dissociation of $(\mathrm{HCl}(3) / 12)$ optimized as described in the text.

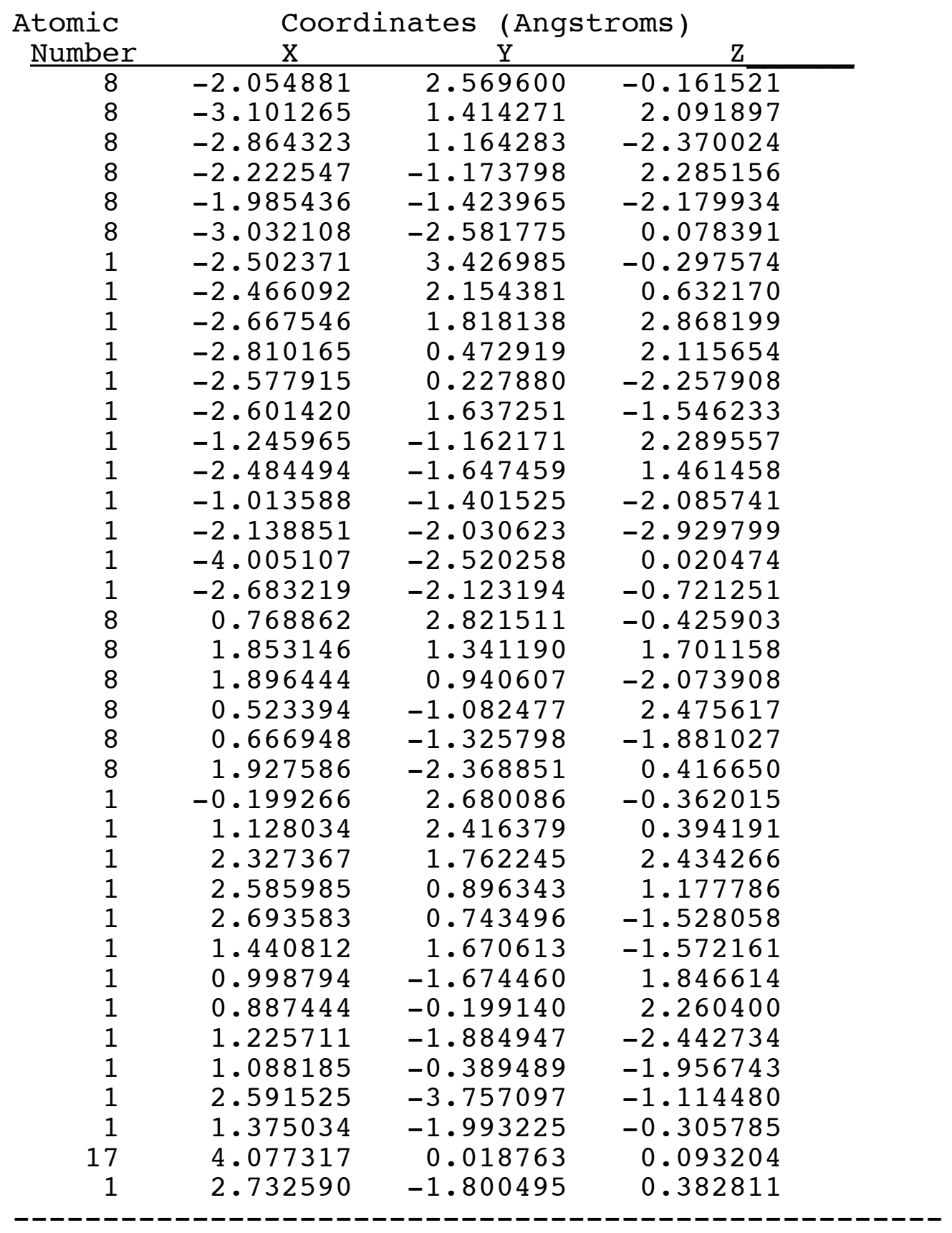


Table S3: Coordinates of $\mathrm{Cl}^{-} .\left(\mathrm{H}_{2} \mathrm{O}\right)_{12}$ anion cluster from dissociation of (HCl(3)/12) optimized as described in the text.

\begin{tabular}{|c|c|c|c|}
\hline Atomic & Coor & (Ang & coms ) \\
\hline Number & $\mathrm{X}$ & $\mathrm{Y}$ & $\mathrm{Z}$ \\
\hline 8 & 1.973467 & 2.581049 & -0.270569 \\
\hline 8 & 3.032356 & 1.097102 & -2.316114 \\
\hline 8 & 2.834104 & 1.576390 & 2.128923 \\
\hline 8 & 2.217901 & -1.507748 & -2.073171 \\
\hline 8 & 2.019508 & -1.028119 & 2.375024 \\
\hline 8 & 3.078712 & -2.515311 & 0.325041 \\
\hline 1 & 2.402240 & 3.458768 & -0.281025 \\
\hline 1 & 2.389233 & 2.050427 & -0.989631 \\
\hline 1 & 2.581463 & 1.357436 & -3.142714 \\
\hline 1 & 2.764905 & 0.158079 & -2.181288 \\
\hline 1 & 2.570657 & 0.627686 & 2.173963 \\
\hline 1 & 2.553421 & 1.900718 & 1.241511 \\
\hline 1 & 1.241077 & -1.519364 & -2.068066 \\
\hline 1 & 2.497645 & -1.832798 & -1.185727 \\
\hline 1 & 1.046580 & -1.044607 & 2.288504 \\
\hline 1 & 2.192818 & -1.501192 & 3.211989 \\
\hline 1 & 4.050438 & -2.421346 & 0.360601 \\
\hline 1 & 2.724131 & -1.939561 & 1.042157 \\
\hline 8 & -0.852864 & 2.824588 & -0.029070 \\
\hline 8 & -1.924740 & 0.984961 & -1.851229 \\
\hline 8 & -1.903974 & 1.185725 & 1.902724 \\
\hline 8 & -0.522487 & -1.503477 & -2.230578 \\
\hline 8 & -0.639638 & -1.057833 & 2.106357 \\
\hline 8 & -1.889803 & -2.500152 & -0.030778 \\
\hline 1 & 0.117020 & 2.691027 & -0.082149 \\
\hline 1 & -1.213257 & 2.281937 & -0.765329 \\
\hline 1 & -2.422106 & 1.257888 & -2.637150 \\
\hline 1 & -2.635783 & 0.609241 & -1.249784 \\
\hline 1 & -2.705577 & 0.888129 & 1.410678 \\
\hline 1 & -1.477270 & 1.843459 & 1.288300 \\
\hline 1 & -0.980359 & -1.988355 & -1.499323 \\
\hline 1 & -0.902454 & -0.604166 & -2.163341 \\
\hline 1 & -1.157173 & -1.457568 & 2.821679 \\
\hline 1 & -1.068566 & -0.128708 & 1.991856 \\
\hline 1 & -1.368039 & -2.034980 & 0.657373 \\
\hline 17 & -4.110841 & -0.106237 & -0.026038 \\
\hline 1 & -2.697080 & -1.943512 & -0.092915 \\
\hline
\end{tabular}


Table S4: Coordinates of DBS of $(\mathrm{HCl}(2) / 12)$ optimized as described in the text.

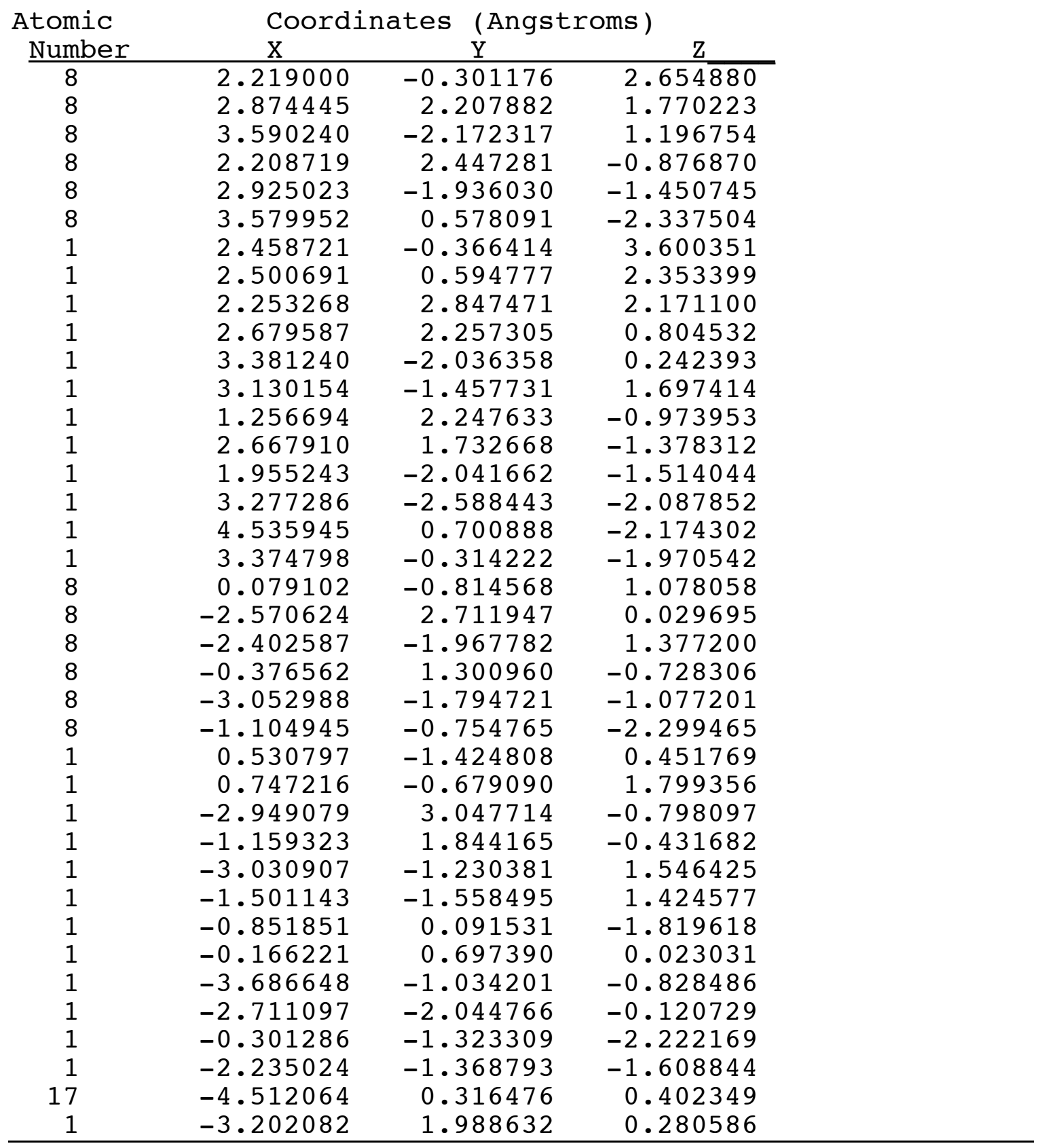


Table S5: Coordinates of $\mathrm{Cl}^{-} .\left(\mathrm{H}_{2} \mathrm{O}\right)_{12}$ anion cluster from dissociation of $(\mathrm{HCl}(2) / 12)$ optimized as described in the text.

\begin{tabular}{|c|c|c|c|}
\hline \multirow{2}{*}{$\begin{array}{l}\text { Atomic } \\
\text { Number }\end{array}$} & \multicolumn{3}{|c|}{ Coordinates (Angstroms) } \\
\hline & $\mathrm{x}$ & $\mathrm{Y}$ & $\mathrm{z}$ \\
\hline 8 & 2.464084 & -0.978840 & 2.364099 \\
\hline 8 & 3.374922 & 1.580194 & 2.004488 \\
\hline 8 & 3.234087 & -2.530547 & 0.241152 \\
\hline 8 & 2.322521 & 2.609323 & -0.306560 \\
\hline 8 & 2.181586 & -1.504337 & -2.071149 \\
\hline 8 & 3.092422 & 1.060165 & -2.431404 \\
\hline 1 & 2.914990 & -1.345702 & 3.148068 \\
\hline 1 & 2.836093 & -0.076313 & 2.224863 \\
\hline 1 & 2.930249 & 2.147472 & 2.662495 \\
\hline 1 & 3.036471 & 1.898238 & 1.134643 \\
\hline 1 & 2.899491 & -2.099982 & -0.580427 \\
\hline 1 & 2.997598 & -1.916262 & 0.975359 \\
\hline 1 & 1.350883 & 2.541271 & -0.244907 \\
\hline 1 & 2.557993 & 1.995331 & -1.041338 \\
\hline 1 & 1.214878 & -1.459688 & -1.944685 \\
\hline 1 & 2.296352 & -1.980173 & -2.915495 \\
\hline 1 & 4.067380 & 1.015989 & -2.427158 \\
\hline 1 & 2.811356 & 0.133944 & -2.243131 \\
\hline 8 & -0.126426 & -0.692600 & 1.346121 \\
\hline 8 & -2.660870 & 2.888940 & 0.133382 \\
\hline 8 & -2.705734 & -1.873029 & 1.593820 \\
\hline 8 & -0.269509 & 1.608603 & -0.228237 \\
\hline 8 & -2.696824 & -2.034118 & -1.163957 \\
\hline 8 & -0.293121 & -0.968378 & -1.377010 \\
\hline 1 & -0.039726 & -1.037868 & 0.424482 \\
\hline 1 & 0.703516 & -0.933565 & 1.809393 \\
\hline 1 & -2.917345 & 3.218831 & -0.741754 \\
\hline 1 & -1.132326 & 2.089579 & -0.107589 \\
\hline 1 & -3.312833 & -1.130624 & 1.381486 \\
\hline 1 & -1.828317 & -1.440927 & 1.682769 \\
\hline 1 & -0.400405 & 0.005657 & -1.264337 \\
\hline 1 & -0.207771 & 0.998220 & 0.541998 \\
\hline 1 & -3.325354 & -1.275605 & -1.152186 \\
\hline 1 & -2.664626 & -2.237622 & -0.193654 \\
\hline 1 & -1.221811 & -1.371290 & -1.393773 \\
\hline 17 & -4.449858 & 0.402612 & -0.047186 \\
\hline 1 & -3.256244 & 2.093689 & 0.229092 \\
\hline
\end{tabular}

Note: Surface is very flat. Optimization stopped at predicted energy change of $\sim 5 \times 10^{-6}$. 